\title{
PREVENTION AND CONTROL OF TUBERCULOSIS
}

\author{
*A.C. Okoh
}

Department of Community Health, University of Teaching Hospital Benin City, Nigeria

\section{Correspondence:}

Dr A. C. Okoh

Department of Community Health, University of Teaching Hospital

Benin City, Nigeria

Email: alexokohmd@yahoo.com

\section{INTRODUCTION}

Tuberculosis is the most frequent cause of death from any infectious agent. It is a leading cause of death in the age group 15-44, especially in women, where it is responsible for $10 \%$ of deaths ${ }^{1}$. About one-third of the world's population is infected, with about 8 million new cases a year occurring between 2003 - 2007, associated with 3 million deaths. It is the cause of about one-third of all AIDS-related deaths in Africa.

For 15 years now, World Health Organization (WHO) declared tuberculosis to be a global emergency. There is an estimated 490,000 new cases of MDR-TB and 30,000 new cases of XDR-TB. Factors that worsen this trend include HIV infection, MDRTB, Tobacco, Prisons, Age, Poverty, etc.

THE CONTROL OF TUBERCULOSIS Control is the reduction of the prevalence and incidence of disease in the community. According to WHO, TB is controlled when natural infection is age 0-14 years is in the order of $1 \%^{2}$. Control, as with other infectious diseases, basically has curative and preventive components. Local government is the operational unit for case detection, chemotherapy, case holding, health education and other activities relating to the National Tuberculosis and Leprosy Control Program (NTBLCP).

Targets for the TB control: To detect at least $70 \%$ of the estimated infectious (smear-positive) cases and to achieve a cure rate of at least $85 \%$ of the detected smear-positive cases.

The 'stop TB' strategy: A program initiated by WHO to stem the tide of TB globally. Health facility and personnel constitute a major drive. This is a control program that has research as one of its control tools ${ }^{2}$.

\section{PRIMORDIAL PREVENTION}

Adopting the proper lifestyle and behavioural changes, especially in early childhood is the basis of primordial prevention. For TB, it will involve the following measures;

- Nutritional interventions

- Environmental modifications (especially healthful housing and proper sanitation) and

- Appropriate health education

\section{PRIMARY PREVENTION}

Specific protection against TB includes; BCG-induces a benign, artificial primary infection which will stimulate an acquired resistance to possible subsequent infection with virulent tubercle bacilli. Also, Chemoprophylaxis .e.g. Intermittent 
Preventive Therapy (IPT) for the immunocompromised.

\section{SECONDARY PREVENTION}

Early diagnosis entails case findings; Diagnostic tools, the cases, suscepts are all involved in early diagnosis as a preventive measure.

Prompt treatment, viz-a-viz, chemotherapy which is currently the mainstay of which is DOTS strategy.

TB control is based on DOTS policy package which includes:

1. Sustained political and financial commitment at all levels of government.

2. Early case detection

3. Diagnosis through quality assured network of TB sputum microscopy services

4. Standardized short course chemotherapy for all cases under proper case management conditions, including direct observation of treatment.

5. Uninterrupted supply of quality of anti-TB drugs

6. Effective monitoring through standardized recording and reporting system.

7. Adequate chemotherapy based on multi drug therapy (MDT).

8. Provision of comprehensive patient care.

\section{TERTIARY PREVENTION}

- Limitation of disability that may result from the disease.

- Rehabilitation- this is needed more in the chronically ill, those still excreting the bacilli mostly need rehabilitation.

\section{- Surveillance}

This involves the continous scrutiny of the factors that determine the occurrence and distribution of disease, TB for effective control and prevention. It involves the collection, analysis, interpretation and distribution of relevant data for action ${ }^{3}$.
An integral part of any effective TB control programme is survelliance. It is in 2 parts;

- Survelliance of the TB situation( i.e annual infection rates)

- Survelliance of control measures applied, e.g BCG, chemotherapy etc ${ }^{4}$.

\section{CONCLUSION}

TB remains a worldwide public health problem despite the fact that the causative organism was discovered over 100 years ago and highly effective drugs and vaccine are available making TB a preventable and curable disease.

However, the perpetuation of 'nonspecific' determinants of disease in third world countries has impeded a rapid conquest of this disease.

\section{RECOMMENDATIONS}

- Rapidly mapping out hot spots using procedures appropriate for outbreak investigations rather than slower methods for standard surveillance.

- Develop new approaches for intensified case finding in high prevalence settings as an adjunctive strategy to DOTS.

- In many high prevalence settings, there is a dire need to strengthen laboratory capacity as few laboratories are equipped to detect drug resistant TB.

- Address practical questions related to the implementation of infection control procedures: how to screen, how to protect healthcare workers and what the efficacy of environmental controls such as ventilation and UV germicidal irradiation is all about.

- Develop effective protocol to roll out IPT in the immunocompromised, bearing in 
mind the risk of Isoniazid resistance.

- Resources, advocacy, and community mobilization to push for implementation and to prioritize additional supporting IPT.

\section{REFERENCES}

1. Murray TJ, Lopez YB. WHO Report global tuberculosis control: Survelliance, planning, financing. Geneva: WHO. 2008: pp20-8.

2. Ludd JJ, Pote DU. Stop TB: A global appraisal. Geneva: WHO. 2007 WHO Bulletin. Available:

http://wwwstopTB.org/tuberculo sis/default.asp

Accessed 9/11/08.
3. Park K. Textbook of Preventive and Social Medicine $18^{\text {th }}$ ed. India M/S Banarsidas Bhanot. 2005: pp33, 83.

4. Bolts SU, Vann BU. Preventive and treatment of tuberculosis among patients with immunodeficiency virus: Principles of therapy and rived recommendations. NY. MMWR. 1998: pp47, 37-41. 